\title{
Preventing sudden cardiac death early after myocardial infarction-ICDs are not the solution
}

Implantation of a cardioverter-

defibrillator in the acute phase after myocardial infarction does not reduce mortality among patients at increased risk for sudden cardiac death. This finding was reported in the New England Journal of Medicine by Gerhard Steinbeck and colleagues of the IRIS study group.

Several randomized trials have demonstrated improvements in survival associated with the use of implantable cardioverter-defibrillators (ICDs) in the settings of life-threatening ventricular arrhythmias (the AVID Trial), myocardial infarction with severe left ventricular dysfunction (MADIT II), and congestive heart failure with reduced ejection fraction (SCD-HeFT). Current guidelines recommend delaying initiation of ICD therapy for at least 40 days after myocardial infarction; however, as Dr Steinbeck highlights "the rate of sudden cardiac death is indeed highest in the very first weeks after a heart attack". In the absence of proven survival benefit for antiarrhythmic drugs, the IRIS investigators set out to determine whether the use of ICDs early after myocardial infarction would significantly reduce the incidence of sudden cardiac death when compared with optimal medical therapy.

The cohort for this study was derived from a registry of 62,944 patients admitted to 92 treatment centers between 1999 and 2007. The patient population was highly selected; those with NYHA class IV heart failure refractory to medical treatment, ventricular arrhythmias before or within $48 \mathrm{~h}$ after myocardial infarction, or indication for CABG surgery were excluded. In addition, two specific inclusion criteria that are markers of increased risk for sudden cardiac death were stipulated-heart rate of $90 \mathrm{bpm}$ or higher with a left ventricular ejection fraction of $40 \%$ or less, and heart rate of $150 \mathrm{bpm}$ with nonsustained ventricular tachycardia. A total of 898 patients met

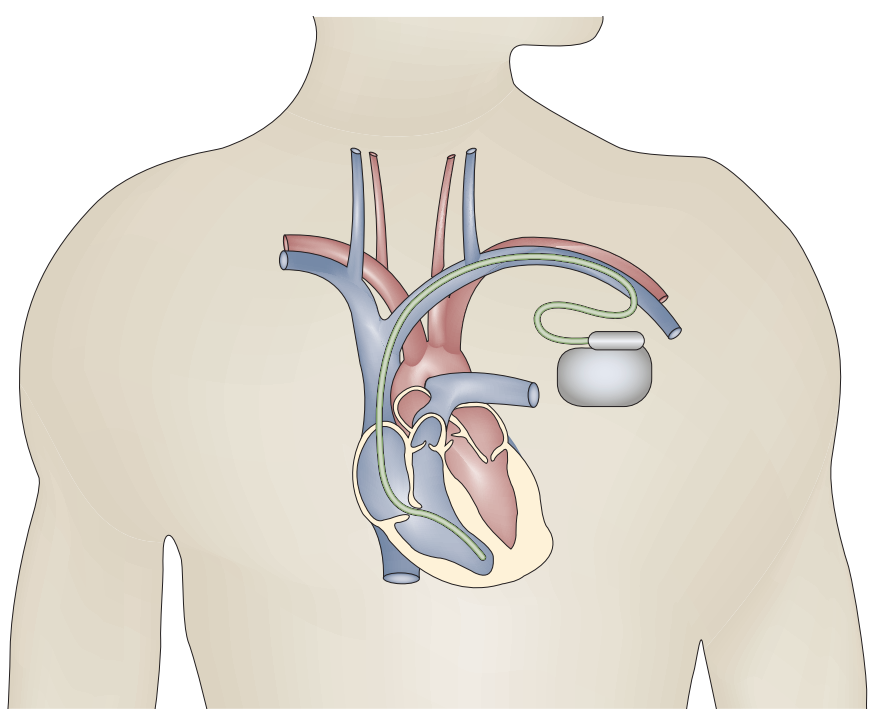

one or both of these criteria, 445 were randomly assigned to receive an ICD (Medtronic, Minneapolis MN) and 453 to medical therapy ( $\beta$-blockers, angiotensinconverting-enzyme inhibitors, statins, and antiplatelet drugs, as prescribed by their physician). The average duration between myocardial infarction and the start of treatment was $13( \pm 7)$ days.

During follow-up (mean 37 months; range $0-106$ months) mortality did not differ significantly between the two treatment groups. Survival at 1, 2, and 3 years was $89.4 \%, 84.6 \%$, and $77.6 \%$, respectively, in the ICD group and $87.5 \%$, $81.8 \%$, and $77.1 \%$ in the medical-therapy group. There was significantly lower mortality from sudden cardiac death among patients who received an ICD, although the benefit was tempered by a higher rate of death from other cardiac causes in this group. These results did not alter significantly when the data were analyzed by subgroup (patients who met either of the inclusion criteria alone, or both of the criteria). Complications necessitating surgical correction of the device, intravenous drug administration, or hospitalization occurred in $15.7 \%$ of patients who received an ICD.
The investigators speculate that the lack of benefit from ICD therapy could have been related to crossover between groups $(10.1 \%$ from ICD to medical therapy and $8.6 \%$ from medical therapy to ICD), inappropriate ICD shocks or other device-related adverse effects, or the use of concomitant therapies. Moreover, they point out that developments in the treatment of myocardial infarction since initiation of the study in the late 1990s could mean that the benefits of ICD therapy in this group of patients are no longer clinically significant.

Looking to the future, "it will be of the utmost importance to identify the cause(s) of the increase in non-sudden cardiac death" says Dr Steinbeck, and there is the possibility that "alternative treatment [such as] externally wearable defibrillator vests, subcutaneous ICDs, and new drugs may better protect patients at risk of dying suddenly in the early phase of myocardial infarction.

Alexandra King

Original article Steinbeck, G. et al. Defibrillator implantation early after myocardial infarction. N. Engl. J. Med. 361, 1427-1436 (2009) 